\title{
Safety and effectiveness of coronary sinus leads extraction - single high-volume centre experience
}

\author{
Andrzej W. Kutarski ${ }^{1}$, Wojciech Jaché́ ${ }^{2}$, tukasz Tułecki ${ }^{3}$, Konrad Tomków ${ }^{3}$, Paweł Stefańczyk ${ }^{4}$, \\ Wojciech Borzęcki ${ }^{4}$, Dorota Nowosielecka ${ }^{4}$, Marek Czajkowski ${ }^{5}$, Maciej Polewczyk ${ }^{6,7}$, Anna Polewczyk ${ }^{6,8}$ \\ ${ }^{1}$ Cardiology Department, Medical University of Lublin, Lublin, Poland \\ ${ }^{2} 2^{\text {nd }}$ Department of Cardiology, School of Medicine with the Division of Dentistry in Zabrze, Medical University of Silesia in Katowice, Poland \\ ${ }^{3}$ Department of Cardiac Surgery, The Pope John Paul II Province Hospital, Zamość, Poland \\ ${ }^{4}$ Department of Cardiology, The Pope John Paul II Province Hospital, Zamość, Poland \\ ${ }^{5}$ Department of Cardiac Surgery, Medical University of Lublin, Lublin, Poland \\ ${ }^{6}$ Department of Medicine and Health Studies, Jan Kochanowski University, Kielce, Poland \\ ${ }^{7}$ Acute Cardiac Care Unit, Swietokrzyskie Cardiology Center, Kielce, Poland \\ ${ }^{8}$ Department of Cardiology, Swietokrzyskie Cardiology Center, Kielce Poland
}

Adv Interv Cardiol 2019; 15, 3 (57): 345-356

DOI: https://doi.org/10.5114/aic.2019.87890

\begin{abstract}
A bstract
Introduction: Transvenous leads extraction (TLE) of permanently implanted coronary sinus (CS) leads is widely believed to present greater risks than the removal of other leads.

Aim: To assess the safety and efficacy of CS leads extraction based on large research material obtained by one operator performing procedures in two TLE centres.

Material and methods: We extracted 408 CS leads from 389 patients, and the results were compared to a control group of 2465 patients who underwent non-CS lead TLE procedures.

Results: There were no significant differences in the clinical success rate $(97.9 \%$ vs. $98.0 \%)$ or the major complication rate $(2.1 \%$ vs. $1.8 \%$ ) between the CS and control group. CS lead destination (LV/LA pacing) and tip location (CS ostium/mid CS /CS tributaries) influenced the procedural and radiological success rates and procedural complexity but not the complications. CS lead extraction did not affect the necessity for a cardiosurgical intervention or presence of procedure-related death.

Conclusions: TLE of CS leads can be achieved with a high procedural success rate. The major complication rate is not higher than that seen in non-CS lead extraction patients. More than half of CS leads cannot be removed by simple traction and the use of mechanical sheaths may be necessary. The detachment of CS leads from connective tissue scars in the venous and atrial areas up to the CS ostium is generally sufficient for further removal of the lead using simple traction.
\end{abstract}

Key words: coronary sinus lead extraction, transvenous lead extraction, cardiac veins.

S u m m a ry

According to previous reports, transvenous extraction (TLE) of coronary sinus (CS) leads may be more risky than in other pacing leads. The present study analysed the biggest group of patients with CS leads undergoing TLE in one centre. The analysis includes CS leads with the longest dwell time. This is also the first description of the feasibility of extraction of leads designed for left atrial pacing (atrial resynchronisation therapy).

\section{Introduction}

The growing number of implanted cardiac resynchronization therapy pacemakers (CRT-P) and defibrillator (CRT-D) systems with a higher number of replacement and upgrade procedures contributes to a frequent necessity for transvenous extraction of the leads (TLE) implanted in the coronary sinus (CS) and cardiac veins designed for left ventricular pacing [1-19]. Over the last

\section{Corresponding author:}

Prof. Anna Polewczyk MD, PhD, Department of Medicine and Health Studies, Jan Kochanowski University, 19 IX Wieków Kielc, 25-317 Kielce, Poland, phone: +48 4136715 08, e-mail: AnnaPolewczyk@wp.pl

Received: 29.12.2018, accepted: 7.04.2019. 
decade, biatrial pacing systems for atrial resynchronisation were implanted using dedicated or non-dedicated leads for permanent left atrial pacing from the CS, and these patients may also require TLE [20-22]. The scale of the problem is reflected by the results of the ELECTRa Registry: among 3555 patients, 755 had a CS lead $(21.2 \%)$ and of the 6493 extracted leads, 640 (10.0\%) were CS leads (547 in CS tributaries (CST) and 140 with other CS location) [23]. From this large registry, it appears that patients with CS leads designed for left atrial pacing represent a negligible percentage. Nowadays atrial resynchronisation therapy is less frequently used; nevertheless, such location of leads is still present and we considered the additional analysis of such a unique group of patients particularly interesting.

\section{Aim}

The risk of CS lead extraction is generally considered to be high, but there are limited data to support this hypothesis. The objective of this study was to analyse the effectiveness and safety of coronary sinus/cardiac vein lead extraction in the biggest population of patients with the longest dwell time of the leads.

\section{Material and methods}

Patient records for this study were obtained from the computer database of the reference centre including information on TLE procedures performed between March 2006 and October 2018.

\section{Patients and procedures}

Records were reviewed for 2854 patients $(60.8 \%$ male), aged 5-94 (66.5 \pm 15.7$)$ years. Clinical characteristics of patients are presented in Table I. Indications for TLE included: systemic infection in $24.2 \%$ of patients, local isolated pocket infection in $10.9 \%$; and non-infective indications in $65.0 \%$ of patients (superfluous non-functional lead, recapture of venous approach, superfluous functional lead, lead posing a potential future threat to the patient if left in place, missed tip location, lead interference, life-threatening arrhythmias secondary to retained lead). The number of leads extracted from individual patients varied from 1 to 6 (mean: $1.66 \pm 0.8$ ). Implantable cardiac defibrillator (ICD) leads were extracted from 767 (26.9\%) patients. CS/CST leads were removed from 389 of the 2854 (13.6\%) patients. The dwell time of the extracted leads ranged from 6 to $386(90.5 \pm 70.4)$ months.

\section{Study group}

The population of patients who had CS or CST leads extracted consisted of 389 subjects divided into 3 subgroups:

I. Patients with left ventricular pacing leads extracted from the cardiac vein system, including all models of leads designed for left ventricle (LV) pacing (only three with active fixation). This subgroup included 187 patients with LV pacing leads extracted from CS branches. Coronary sinus branch/tributary (CST) permanent pacing leads used for LV pacing, for ventricular resynchronisation (187 patients) in the years 1999-2017 were: Biotronik leads - Corox LVP 75, Corox LV S 75 and Corox OTW different models (56 patients); later on our markedly predominant Medtronic leads - mainly Attain UP and BP and a large assortment of subsequent models of Attain OTW models (116 patients); SJM (Saint Jude Medical) leads (different models of QuickFlex) were not that common (15 patients). About $75 \%$ of the LV leads were bipolar or quadripolar. Two leads from cardiac veins were extracted from 1 patient.

II. Patients with left atrial CS leads extracted from the mid CS (CSM), including non-dedicated passive, standard bipolar leads and dedicated passive bipolar leads. This subgroup included 116 patients who had left atrial (LA) pacing leads for atrial resynchronisation extracted (116 patients). They were implanted in 2002-2005 with leads dedicated for LA pacing as Corox LA (CX55, Cx63) and experimental models (V182, V202, V375, V322) - 40 patients. Other leads not dedicated for LA pacing - standard BP leads (with one or two tines removed, for better contact of a lead tip with the CS wall) - were passive BB BP standard leads, such as TIR 60, which were implanted in 1996-2002 in a pioneer era of biatrial pacing - 27 patients. In some patients active fixation leads were implanted in the mid/proximal part of the CS. There were Biotronik leads such as Y 60 BP or RX (Retrox) 60 BP or later Elox 53 BP, Selox $53 \mathrm{BP}$ in 49 patients. Two leads from the mid CS were extracted from 4 patients (Figure 1).

III. Patients with (LA) coronary sinus ostium (CSO) leads extracted, including non-dedicated straight screw-in leads. This subgroup consists of 86 patients whose leads were extracted (2 leads were extracted from 12 patients). CSO permanent pacing for LA pacing for atrial resynchronisation (86 patients) was utilised in the years 2004-2008. Standard active fixation leads were implanted, usually Biotronik leads, such as Elox 53 BP, Selox 53 BP, or Setrox 53 BP in 71 patients and rarely $Y 60 \mathrm{BP}$ or Tendril BP (SJM) - in 15 patients

\section{Control group}

The remaining 2465 patients served as the control group.

\section{Definitions}

All definitions used in this study for lead removal/ extraction, clinical and radiological success, and complications were in accordance with the 2017 Heart Rhythm Society Expert Consensus and 2018 EHRA Expert Consensus on Lead Management and Extraction [24, 25]. 

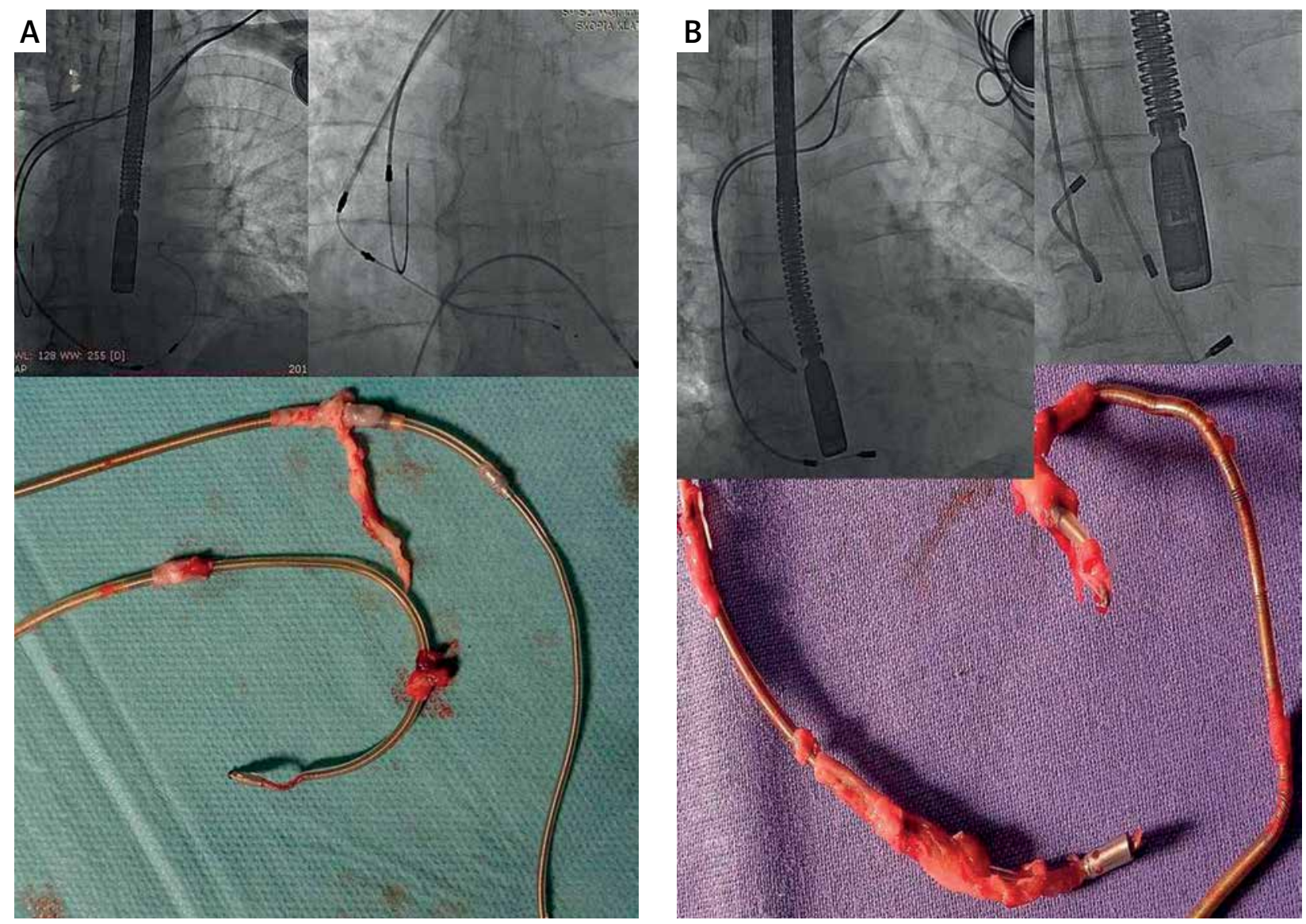

Figure 1. Extraction of CS lead designed for permanent LA pacing for atrial resynchronisation. Strongest connective tissue scar near CS ostium (around anodal ring of the lead), lack of connective tissue remnant on the anchoring strand (A) and extraction of CSO screw-in lead designed for permanent LA pacing for biatrial pacing. Entrance with Byrd dilator into proximal CS was necessary to liberate both of the electrodes from the connective tissue scar. Strongest solid scar around anodal ring of the lead (B)

\section{Lead extraction standard procedures}

TLE procedures were performed using mechanical cutting-rotation force with telescopic polypropylene Byrd dilators (all sizes and lengths; Cook Medical, Bloomington, $I N)$, generally via the subclavian approach (lead venous entry). Laser energy and radiofrequency waves were not used. Screw-in leads with a dwell time of $\leq 2$ years were removed, if possible, by simple extorsion and gentle traction only (but only in infectious cases). In case of a lead replacement we favoured the use of mechanical dilatation to keep the same venous entry approach for the new lead. Spontaneously broken leads with the proximal end dropped into the cardiovascular system and broken lead fragments were extracted using a femoral, right jugular, or other recaptured lead venous entry approach.

\section{CS lead extraction}

In infectious cases, lead removal by simple traction was attempted. When noticeable resistance was encountered, polypropylene Byrd dilators, as thin as possible (internal diameter of internal sheaths: 7.0 Fr-blue, 8.5 Fr- yellow or 10.0 Fr-green as a last resort), were used. If CSO or CST lead replacement was necessary, venography was conducted. When vein occlusion was suspected, indicating potential problems with a new lead implantation, the procedure was started with Byrd dilators to maintain the venous access. We noted various degrees of difficulty of CSM/CST lead extraction, depending on the lead construction and dwell time.

\section{Statistical analysis}

Statistical analyses were carried out using Statistica v. 12.0 (StatSoft, Tulsa, OK). Means and standard deviations (SDs) were calculated for continuous variables, whereas qualitative variables were presented as absolute and relative (percentage) quantities. An unpaired Student's $t$-test was used to compare 2 quantitative variables. Pearson's $\chi^{2}$ test was used to compare qualitative variables, whereas Yates' $\chi^{2}$ test was used for small samples ( $<5$ subjects). $P<0.05$ was considered statistically significant. To evaluate the relationship between the variables and the radiological success of TLE and de- 
velopment of major complications multivariable logistic regression analysis was performed. The Kaplan-Meier method was used to calculate the probability of living through the period of time free of events depending on lead location and destination (CST, CSM, CSO) and the log-rank test, including complete and censored data, was used to test for differences between the survival curves. A two-tailed $p$-value $<0.05$ was considered statistically significant.

\section{Results}

CS leads extractions among all TLE procedures - the scale of the problem

Procedure analysis showed that among 2854 TLE, in 389 patients CS leads were extracted (13.6\%). Lead analysis demonstrated that of the 4729 extracted leads, 408 (8.6\%) were CS leads (188 in CS tributaries and 220 with other CS location) (Table I).

\section{Patient analysis}

The CS (any) patient population consisted of 2 different clinical groups: patients with heart failure and ventricular resynchronisation (LV leads) and patients with brady-tachycardia syndrome, a severe interatrial block, treated with atrial resynchronisation (mid CS and CS ostium subgroups). Patients with CRT systems (CS tributary located leads) were younger, more often male, and presented more often with renal failure, diabetes, poor NYHA class, lower left ventricular ejection fraction (LVEF) and higher Carlson's index in comparison to the control group (without CS leads) and with both LA pacing (atrial resynchronisation) groups. Patients with brady-tachycardia syndrome and atrial resynchronisation were older, more often female, and had less frequently renal failure, diabetes, poor NYHA class, lower LVEF, lower Carlson's index and more points on the $\mathrm{CHA}_{2} \mathrm{DS}_{2}$-VASc scale in comparison to the control group (without CS leads) and patients with CRT generally. There were some differences among the CS subgroups and the control group in indications for lead extraction. In patients with CRT systems (CST leads) lead extraction due to infectious indications was performed much more frequently.

\section{System-related factors}

Patients with CRT systems (CST leads) presented a significantly shorter lead dwell time and had more cardiac implantable electronic devices (CIED) related procedures before the lead extraction, more leads in the system, more leads extracted in one patient during the procedure and more ICD leads extracted in comparison to the control group and two subgroups with LV leads (Table I).

Patients with brady-tachycardia syndrome and atrial resynchronisation (CMS and CSO groups) presented a significantly longer lead dwell time and had fewer
CIED-related procedures before the lead extraction, fewer leads in the system, more leads extracted in one patient during the procedure and fewer ICD leads extracted in comparison to the control group and subgroups with LV leads (Table I).

\section{Analysis of TLE procedures}

We removed 408 leads in 389 patients implanted into the coronary sinus (CSO, CSM) or via the coronary sinus into the cardiac vein (CST). In 19 patients two CS leads were extracted.

\section{Left ventricular (CST) lead extraction}

We removed $188 \mathrm{LV}$ leads in 187 patients. In 1 patient we extracted two LV leads. Sixty-eight CST leads (36.4\%) were removed with simple traction only. For extraction of the remaining 119 CST leads (63.6\%) we used polypropylene Byrd dilators due to strong connective tissue or to keep (gain) a venous approach in case of lead replacement.

\section{Mid CS (CSM) lead extraction}

We removed 122 leads from the mid CS in 116 patients. In 6 patients we removed two leads. Only 12 CMS leads $(9.8 \%)$ were removed with simple traction. For extraction of the remaining 110 CMS leads (90.2\%) we used Byrd dilators due to a strong scar or to maintain/ recover a venous approach in case of a necessity for lead replacement. In 4 (3\%) patients, the use of a combined approach was necessary, but not due to the need for lead detachment in its CS part.

\section{Coronary sinus ostium (CSO) lead extraction}

We removed 98 leads from the CS ostium (CSO) in 86 patients. In 12 patients two CSO leads were extracted. Four CSO leads (4.0\%) were removed with simple traction only. For extraction of the remaining 94 CSO leads (95.9\%) we used polypropylene dilators due to connective tissue surrounding the lead or with the intention to retain or to render possible a venous approach for new lead implantation. In 1 (1\%) patient the use of a combined approach was necessary but not due to the necessity of a lead detaching in its CS part.

\section{Technical aspects of TLE in CS population}

Over $50 \%$ of the LV leads (CST) could be removed by simple traction but in cases of venous stenosis we tried to pass with a polypropylene sheath over the lead to the superior vena cava with the intention of keeping a venous approach for a new lead. Nearly $90 \%$ of the CSM (LA) passive leads had to be extracted with Byrd dilators, which was probably related to their long mean dwell time of 110 months (9.2 years). Only $4 \%$ of the screw-in leads located in the proximal CS could be removed by a simple 


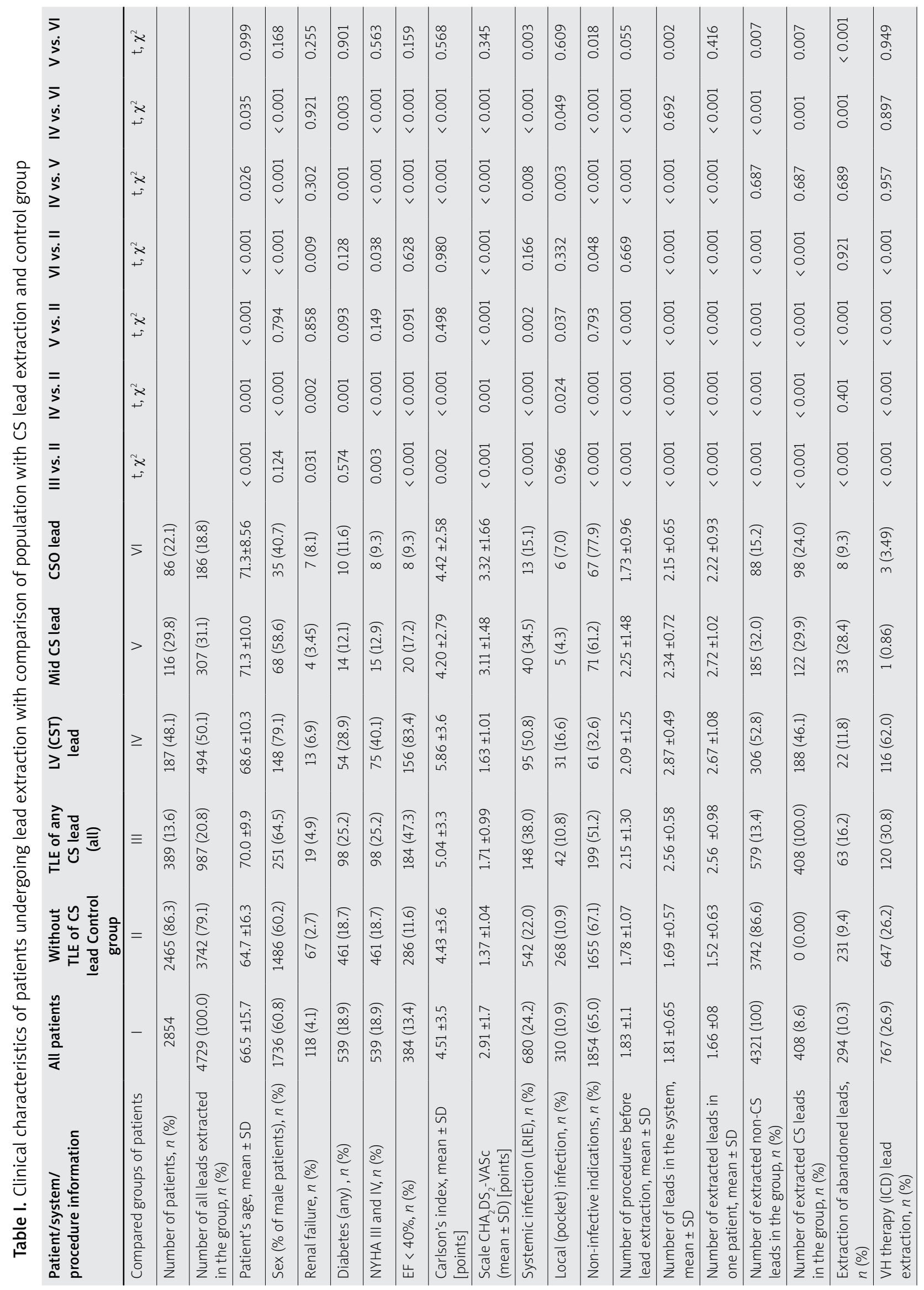




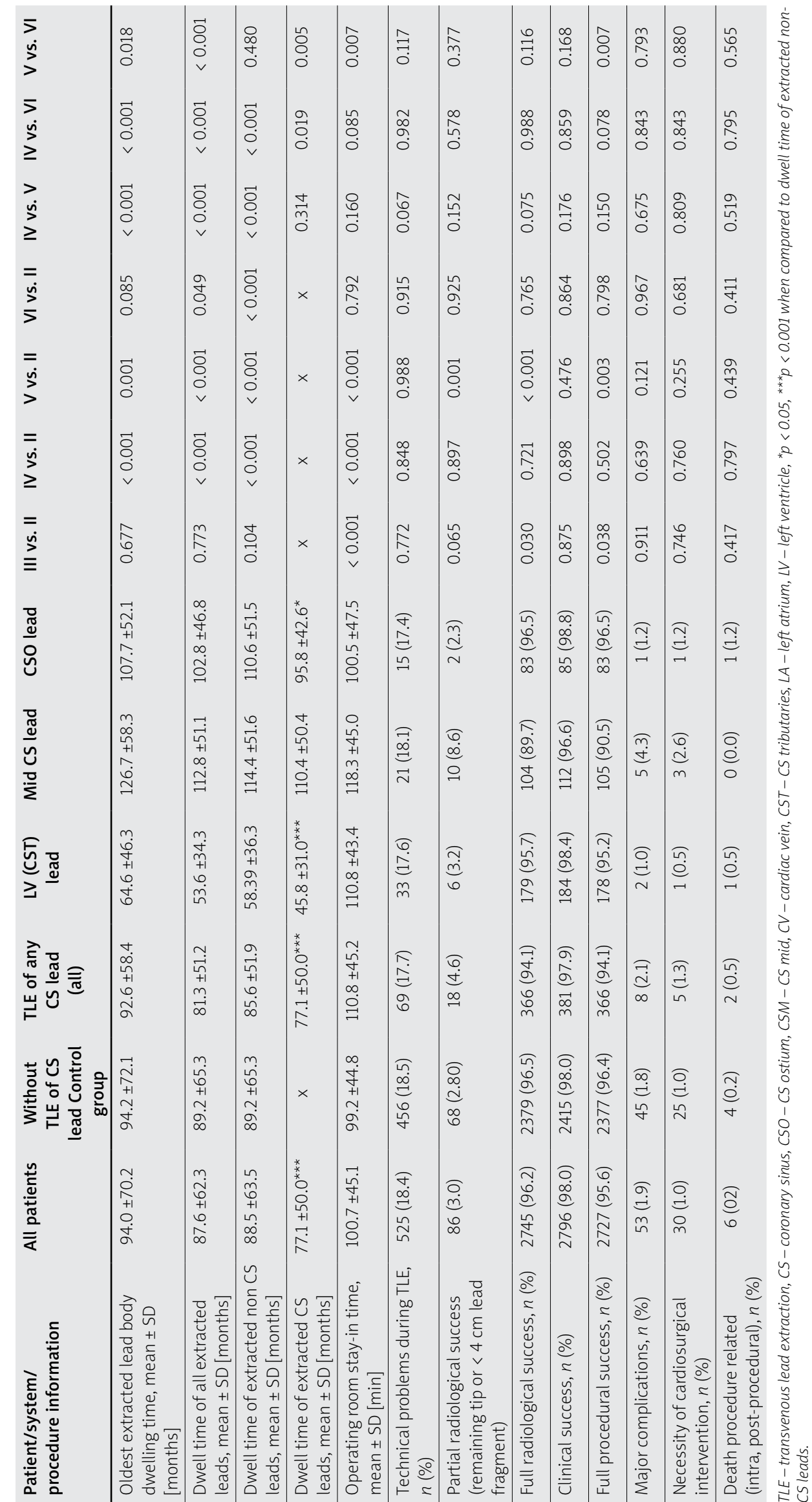



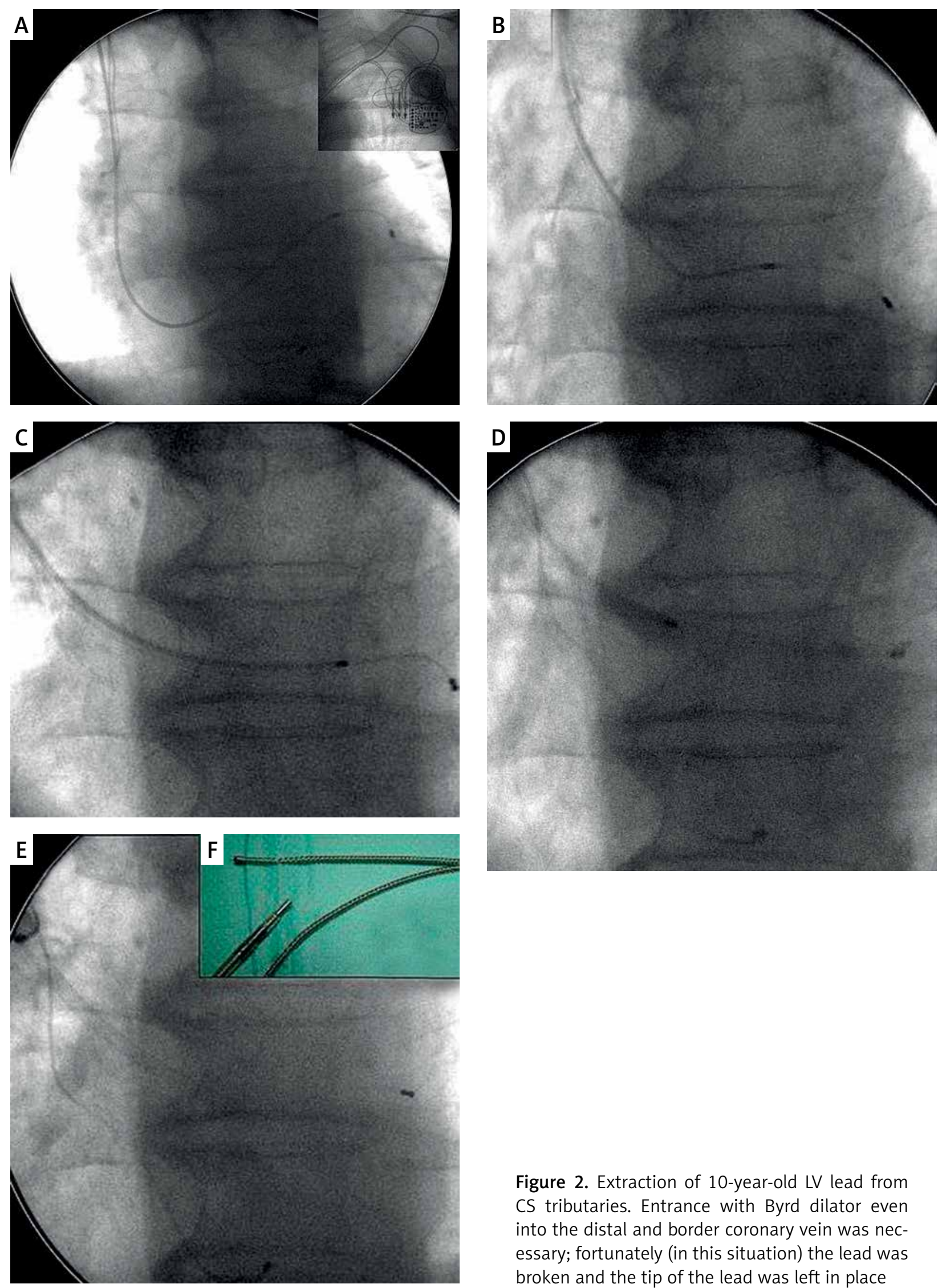

Figure 2. Extraction of 10-year-old LV lead from CS tributaries. Entrance with Byrd dilator even into the distal and border coronary vein was necessary; fortunately (in this situation) the lead was broken and the tip of the lead was left in place 
screw-out and gentle traction; for $>90 \%$ of such leads mechanical sheaths were necessary even though most of the screw-in bipolar leads were isodiametric. Again, this might be explained by the average implant dwell time of 95.8 months.

During only 5 of 188 LV (CST) bipolar lead extractions $(2.7 \%)$ it was necessary to introduce a mechanical sheath up to the mid-CS, and similarly for 6 of 122 (4.9\%) passive bipolar leads located in the CS and designed for LA pacing. In these leads it was sufficient to liberate the proximal ring to remove the entire lead from the body. When the introduction of a Byrd dilator inside the CS was necessary, we used only a single internal sheath, as thin as possible (usually the yellow 8.5-Fr sheath). In two cases, for the detachment of an LVV lead in the CS tributary region we used a CS lead introducing the sheath using the Bongiorni technique [9]. In 1 patient a broken LV (CST) lead fragment $(1.5 \mathrm{~cm})$ was left in the cardiac vein and in an additional 2 patients a distal part of the CS lead (broken anchoring strand) $-<4 \mathrm{~cm}$ fragment - was left in the CS (Figure 2).

\section{Analysis of effectiveness and safety of TLE procedures}

Clinical success (lead extraction without major complications or death) was obtained for $97.9 \%$ of patients with any CS lead and it was comparable in all analysed groups (Table I). Full radiological success was achieved in

Table II. Multivariate analysis of factors affecting the radiological and clinical success of TLE and factors affecting the occurrence of major complications

\begin{tabular}{|c|c|c|c|}
\hline Parameter & OR & $95 \% \mathrm{Cl}$ & $P$-value \\
\hline \multicolumn{4}{|c|}{ Factors affecting the radiological success of transvenous lead extractions, results of multivariable linear regression analysis: } \\
\hline Abandoned lead presence [yes/no] & 0.641 & $0.386-1.065$ & 0.086 \\
\hline Patient's age during TLE [by 1 year] & 1.024 & $1.013-1.035$ & 0.000 \\
\hline Female gender [yes/no] & 0.896 & $0.614-1.309$ & 0.571 \\
\hline NYHA class [by one] & 0.949 & $0.702-1.283$ & 0.732 \\
\hline Prior sternotomy [yes/no] & 0.696 & $0.433-1.118$ & 0.134 \\
\hline Diabetes [yes/no] & 1.590 & $0.859-2.943$ & 0.140 \\
\hline Creatinine concentrations [1 mg\%] & 0.986 & $0.862-1.128$ & 0.842 \\
\hline TLE of ICD lead [yes/no] & 1.886 & $1.039-3.422$ & 0.037 \\
\hline TLE of CS lead [yes/no] & 0.510 & $0.296-0.879$ & 0.015 \\
\hline Number of procedures before lead extraction [by 1] & 0.812 & $0.691-0.954$ & 0.011 \\
\hline Number of extracted leads in one patients [by 1] & 1.481 & $1.069-2.053$ & 0.018 \\
\hline Sum of dwell time of all extracted leads [by 1 year] & 0.956 & $0.939-0.974$ & 0.000 \\
\hline \multicolumn{4}{|c|}{ Factors affecting the occurrence of major complications of transvenous lead extractions, results of multivariable linear regression analysis } \\
\hline Abandoned lead presence [yes/no] & 1.240 & $0.543-2.829$ & 0.609 \\
\hline Patients age during TLE [by 1 year] & 1.002 & $0.981-1.023$ & 0.870 \\
\hline Female gender [yes/no] & 3.833 & $1.948-7.543$ & 0.000 \\
\hline NYHA class [by one] & 0.821 & $0.496-1.358$ & 0.443 \\
\hline Prior sternotomy [yes/no] & 0.705 & $0.243-2.047$ & 0.520 \\
\hline Diabetes [yes/no] & 1.011 & $0.429-2.385$ & 0.980 \\
\hline Creatinine concentrations [1 mg\%] & 1.051 & $0.903-1.222$ & 0.522 \\
\hline TLE of ICD lead [yes/no] & 0.874 & $0.312-2.448$ & 0.798 \\
\hline TLE of CS lead [yes/no] & 1.264 & $0.512-3.120$ & 0.611 \\
\hline Number of procedures before lead extraction [by 1] & 1.280 & $1.004-1.631$ & 0.046 \\
\hline Number of extracted leads in one patients [by 1] & 0.748 & $0.444-1.258$ & 0.273 \\
\hline Sum of dwell time of all extracted leads [by 1 year] & 1.055 & $1.027-1.083$ & 0.000 \\
\hline
\end{tabular}


a similar percentage in both the CS and control group of patients ( 94.1 vs. $96.5 \%$ ); the tip of the lead remained in $1.8 \%$ of patients, a lead fragment remained in $4.1 \%$ of patients. The frequency of clinical success was limited by the appearance of a major complication, whereas the procedural success rate was limited by a remaining, irremovable lead fragment $(96.4 \%$ vs. $95.6 \%$ in compared groups).

The occurrence of technical problems (a mutual lead connection with a strong connective tissue scar, a lead breakage during the extraction, a BP lead fragmentation and a further removal in two parts, a significant blockage in the subclavian lead venous entry, damage of a polypropylene sheath, an unexpected dislodgement of a functional lead, a lead breakage and a loss of a distal fragment free floating/wandering in the cardiovascular system) during lead extraction represents the scale of extraction difficulties. We noted this phenomenon with the same frequency in the CS and the control group (17.7\% vs. $18.5 \%$ ). The procedure duration was slightly longer for patients with CS leads, possibly related to the number of extracted leads (110.8 vs. $99.2 \mathrm{~min}$ ) (Table I).

Major complications occurred in 8 patients in the total (389) CS lead group (2.1\%) and in 45 among 2465 patients in the control group (1.8\%). In the CS group among major complications there were: hemopericardium with tamponade and cardiac surgery $4(1.0 \%)$, hemothorax - drainage $1(0.3 \%)$, pulmonary embolism requiring surgery $1(0.3 \%)$ and TLE-related tricuspid valve dysfunction $2(0.5 \%)$. Procedure-related death (intra- and post-procedural) occurred in $2(0.5 \%)$ cases in the total CS group and in $6(0.2 \%)$ in the control group (Table I).

Multivariable logistic regression analysis showed that the factors of higher probability of achieving radiological success were: older age of the patient (increase by $2.4 \%$ with each year), TLE of ICD lead (increase by $88.6 \%$ ), number of extracted leads (increase by $48.1 \%$ per lead). The risk factors for not achieving radiological success were TLE of abandoned lead (probability lower by $35.9 \%$; $p=0.086$ ), TLE of CS lead (probability lower by $49.0 \%$ ), number of previously procedures (probability lower by 18.8 per procedure) and sum of dwell time of extracted leads (probability lower by $4.4 \%$ per year) (Table II).

The risk factors of major complications were: female gender (more than 3.8 times higher risk), number of previously procedures (increase by 28.0 per procedure) and sum of dwell time of extracted leads (increase by $5.5 \%$ per year) (Table II).

\section{Long-term survival after transvenous lead} extraction in relation to CS lead tip location

Mean follow-up was $5 \pm 3.05$ years. Kaplan-Meier curves showed that long-term mortality after TLE was high and differed in patients regarding their CS lead location and (indirectly) indication for implantation

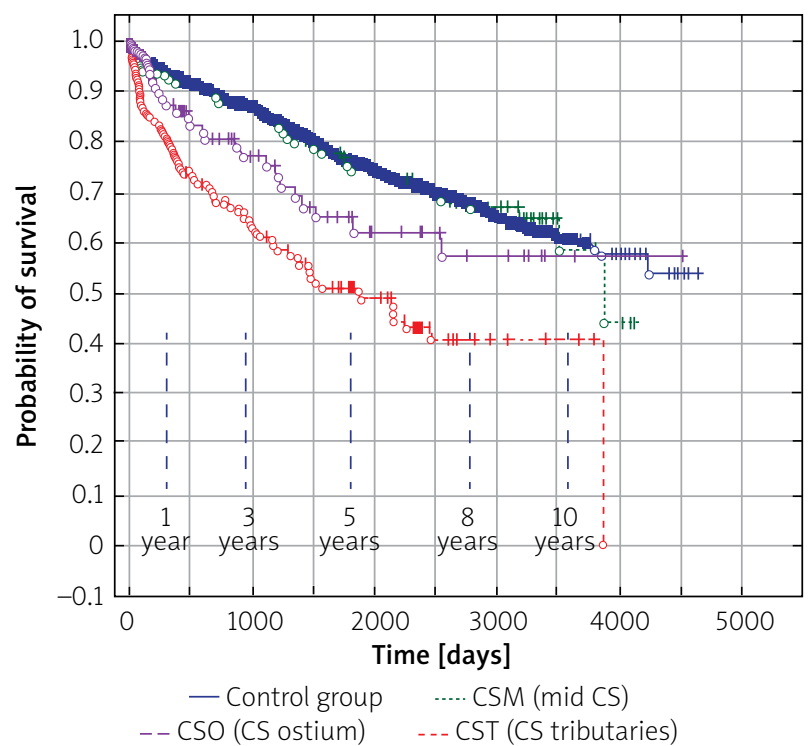

Figure 3. Long-term survival of patients after CS lead location. Kaplan-Meier curves show that long-term mortality differed in patients regarding their CS lead location and (indirectly) indication for implantation. Long-term mortality was higher when the CS lead was located in the CST and the lead served for ventricular resynchronisation (patients with impaired LV function)

( $p<0.001)$ (Figure 3). Long-term mortality was higher when a CS lead was located in CST and a lead served for ventricular resynchronisation (patients with impaired LV function). Mortality of patients having a CS lead for atrial resynchronisation varied between CST and the control group. Fifty percent survival in patients after CST (LV lead) extraction after 5 years FU seems to be optimistic for patients with a high mean Carlson's index (Figure 3).

\section{Discussion}

Cardiac resynchronization therapy, including ventricular and atrial resynchronization (atrial resynchronization therapy), may be associated with the need for LV or LA lead extraction from the CST or CS due to infection, lead malfunction, or system upgrade. The literature on this topic is limited [1-19]. The experience from a low $(<50$ TLE procedures) [7-19] and a little higher ( $>50$ procedures) number of patients has indicated that recently implanted leads (<24 months) can be removed using simple gentle traction [1-6]. For CST leads with a longer dwell time, the use of various sheaths (mechanical or delivering energy) for detachment from connective tissue scars up to the CS ostium (CSO) is sufficient in most cases.

Although most of the current reports are based on small clinical trials, several high volume studies have appeared in recent years [1-6]. The mean dwell time of the LV leads (CST) was 35.7 months [1-19], the procedural success 


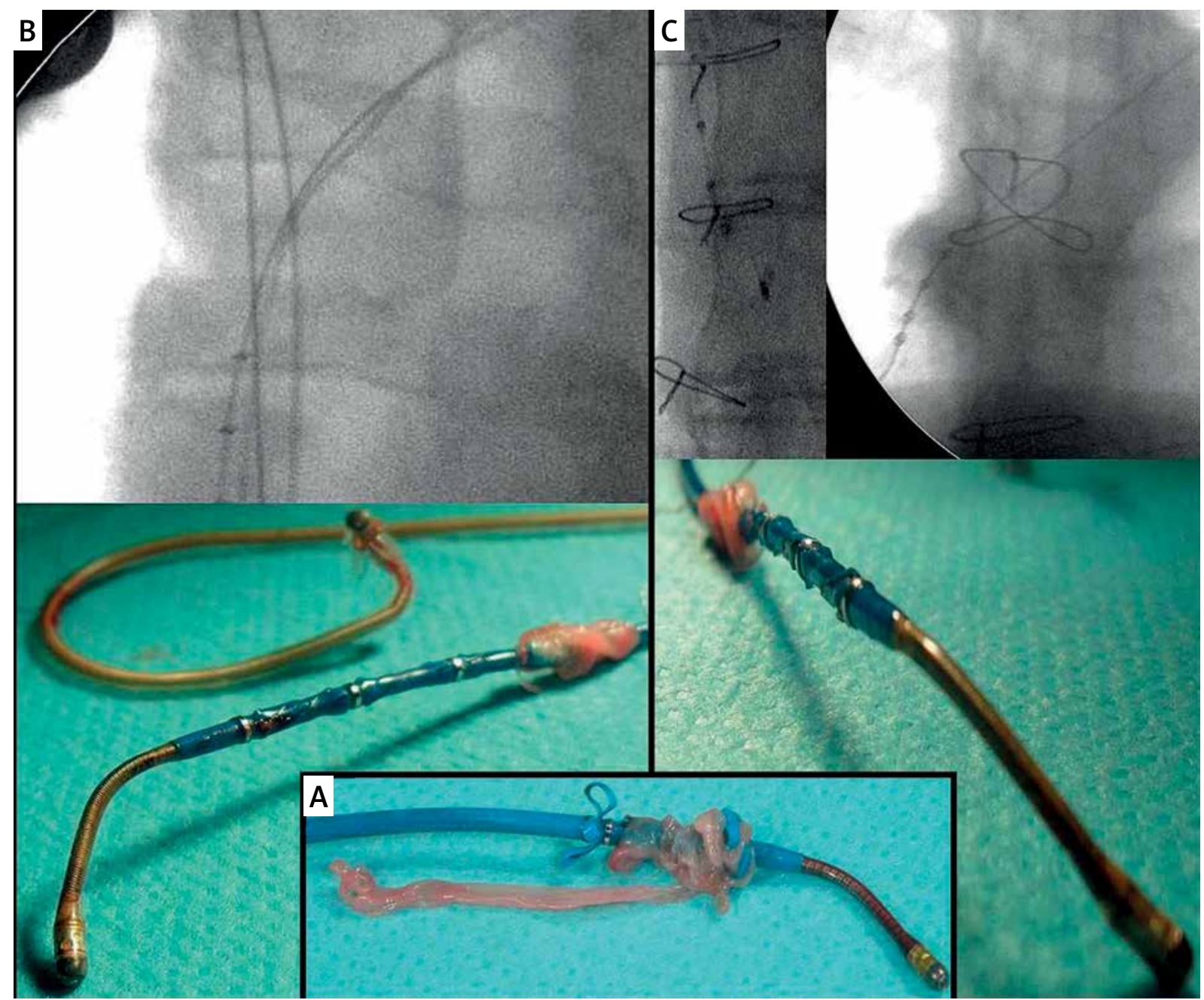

Figure 4. Extraction of StarFix lead (A) by applying tension to the blue fixating external lead sheath, which caused straightening of the 'tines', enabling detachment from connective tissue scar in the cardiac vein (B and C). Use of locking stylets for such leads seems to be contraindicated; the entire tension force has be concentrated on the blue external sheath of the StarFix lead

rate was $98-100 \%$, and major complications occurred in $0-7.5 \%$ of the patients. Most leads could be removed using manual traction/locking stylets (range: 0-100\%, average $66.9 \%$ ); however, difficulties with extraction increased significantly with the dwell time. Such a high success rate using simple traction is not surprising, as 15 years ago, most LV leads were thin, unipolar, and largely isodiametric, without a steroid bulb. Later, the market was dominated by bipolar (and increasingly, multipolar), steroid-eluting leads with a smaller diameter, which were also fairly isodiametric. However, a bulging pacing ring and a protruding lead shadow, designed to improve pacing conditions, may obstruct lead removal by simple traction. The construction of leads designed for LV pacing from the CST should be initiated to facilitate future lead removal.

A challenge for lead explantation is the active fixation LV lead (StarFix; Medtronic, Minneapolis, MN) [1, 4, 15, 19]; after several months, gentle continuous traction may become ineffective and a conventional sheath or modified (soft distal ending) CS lead delivery sheath may be required. We extracted three StarFix leads by applying tension to the blue fixating external lead sheath, which caused straightening of the 'tines', enabling detachment from a connective tissue scar in the cardiac vein. Cited reports characterise the extraction of this lead as more challenging [1], with a higher procedural failure rate compared to passive fixation [13], and a frequent necessity for the use of a mechanical extraction sheath up to $50-75-100 \%[4,15,19]$. Therefore the extraction of such leads should be performed in high-volume extraction centres, with experienced operators and with an on-site cardiac surgery standby $[1,4,13,15,19]$. In our experience, the use of locking stylets for such leads seems to be contraindicated; the total tension force has to be concentrated on the blue external sheath of the StarFix lead.

We have a strong impression that lead construction has a significant influence on CS lead extraction. Lead polarity in older models was important, with easier UP 
lead extraction. Longevity, bulging and blandness of the anodal ring is important especially in large/middle diameter leads. Leads having a porous ring surface (Biotronik) or a spiral metal coil surface (some Medtronic models) were more difficult to extract and such leads needed the CS ostium to be crossed with a mechanical sheath for detachment.

We were unable to locate any previous reports of the extraction of CS leads for permanent LA pacing from the coronary sinus for comparison to our results. Atrial resynchronization is still used in some centres for patients with severe interatrial conduction disturbances and atrial arrhythmias, especially for patients ineligible for ablation or after aggressive atrial fibrillation ablation. Currently, we use 2 screw-in leads positioned in the Bachmann bundle region and in the CSO, but more than 15 years ago a large number of passive leads (dedicated and non-dedicated) were implanted, even into the distal CS [20-22]. The life expectancy of patients with atrial resynchronization is longer than those with CRT systems, and over the years we have extracted numerous LA pacing leads from the CS. Our measured success and complication rates for CS TLE were no higher than in other lead extraction procedures, but our personal impression is that it is still a challenge even for experienced operators.

We have also observed that the connective tissue surrounding the lead in the CS and CV is softer and weaker compared to that in the great veins, atrial walls, and even the CSO. We have never encountered calcification in this portion of the extracted lead. The introduction of mechanical sheaths into the CS is rarely necessary (11/414 leads, $2.6 \%)$, but we do not have CS TLE-dedicated sheaths. Such sheaths may be useful when CS leads liberated with standard sheaths from scars up to the CSO cannot be removed with simple traction and the introduction of a sheath into the CS lumen is necessary. Similar to Byrd dilators, longer, for a femoral approach, but with a more flexible distal part, these sheaths could be very useful in such TLE procedures (Figure 4).

\section{Conclusions}

Transvenous removal of CS leads can be achieved with a high procedural success rate. Major and minor complication rates are no higher than those in non-CS lead extraction patients. Half of the CS leads cannot be removed by simple traction and the use of mechanical (polypropylene) sheaths may be necessary. Detachment of CS leads from connective tissue scars in the venous and atrial portion up to the CSO is sufficient for further removal by simple traction. The connective tissue surrounding the lead in the CS appears to be softer and weaker than that in the great veins, atrial walls, and CSO. Therefore the introduction of a mechanical sheath into the CS lumen is rarely necessary. However, the develop- ment of special sheaths (more flexible in the distal part and longer for a femoral approach) designed for CS lead extraction would be useful in these cases.

The main limitation of the present study was its retrospective design; therefore it was impossible to make direct comparisons between three different sui generis patient groups and analysis of complications associated with the extraction or absence of CS leads.

\section{Conflict of interest}

The authors declare no conflict of interest.

\section{References}

1. Crossley GH, Sorrentino RA, Exner DV, et al. Extraction of chronically implanted coronary sinus leads active fixation vs passive fixation leads. Heart Rhythm 2016; 13: 1253-9.

2. Rickard J, Tarakji K, Cronin E, et al. Cardiac venous left ventricular lead removal and reimplantation following device infection: a large single-center experience. J Cardiovasc Electrophysiol 2012; 23: 1213-6.

3. di Cori A, Bongiorni MG, Zucchelli G, et al. Large, single-center experience in transvenous coronary sinus lead extraction: procedural outcomes and predictors for mechanical dilatation. Pacing Clin Electrophysiol 2012; 35: 215-22.

4. Cronin EM, Ingelmo CP, Rickard J, et al. Active fixation mechanism complicates coronary sinus lead extraction and limits subsequent reimplantation targets. J Interv Card Electrophysiol 2013; 36: 81-6.

5. Sheldon S, Friedman PA, Hayes DL, et al. Outcomes and predictors of difficulty with coronary sinus lead removal. J Interv Card Electrophysiol 2012; 35: 93-100.

6. Williams SE, Arujuna A, Whitaker J, et al. Percutaneous lead and system extraction in patients with cardiac resynchronization therapy (CRT) devices and coronary sinus leads. Pacing Clin Electrophysiol 2011; 34: 1209-16

7. Lisy M, Schmid E, Kalender G, et al. Coronary sinus lead extraction in CRT patients with CIED-related infection: risks, implications and outcomes. Minerva Cardioangiol 2015; 63: 91-8.

8. Lisy M, Kornberger A, Schmid E, et al. Application of intravascular dissection devices for closed chest coronary sinus lead extraction: an interdisciplinary approach. Ann Thorac Surg 2013; 95: 1360-5.

9. Bongiorni MG, Zucchelli G, Soldati E, et al. Usefulness of mechanical transvenous dilation and location of areas of adherence in patients undergoing coronary sinus lead extraction. Europace 2007; 9: 69-73.

10. Hamid S, Arujuna A, Khan S, et al. Extraction of chronic pacemaker and defibrillator leads from the coronary sinus: laser infrequently used but required. Europace 2009; 11: 213-5. Erratum in: Europace. 2009; 11: 538.

11. Starck CT, Caliskan E, Klein H, et al. Results of transvenous lead extraction of coronary sinus leads in patients with cardiac 4,703 resynchronization therapy. Chin Med J 2013; 126: 4703-6.

12. Chu XM, Li XB, Zhang P, et al. Percutaneous extraction of leads from coronary sinus vein and branch by modified techniques. Chin Med J 2012; 125: 3707-11.

13. Pecha S, Kennergren C, Yildirim Y, et al. Coronary sinus lead removal: a comparison between active and passive fixation leads. PLoS One 2016; 11: e0153651. 
14. Kasravi B, Tobias S, Barnes MJ, Messenger JC. Coronary sinus lead extraction in the era of cardiac resynchronization therapy: single center experience. Pacing Clin Electrophysiol 2005; 28: 51-3.

15. Tyers GF, Clark J, Wang Y, et al. Coronary sinus lead extraction. Pacing Clin Electrophysiol 2003; 26: 524-6.

16. Maytin M, Carrillo RG, Baltodano P, et al. Multicenter experience with transvenous lead extraction of active fixation coronary sinus leads. Pacing Clin Electrophysiol 2012; 35: 641-7.

17. De Martino G, Orazi S, Bisignani G, et al. Safety and feasibility of coronary sinus left ventricular leads extraction: a preliminary report. J Interv Card Electrophysiol 2005; 13: 35-8.

18. Burke MC, Morton J, Lin AC, et al. Implications and outcome of permanent coronary sinus lead extraction and reimplantation. J Cardiovasc Electrophysiol 2005; 16: 830-7.

19. Kypta A, Blessberger H, Saleh K, et al. Removal of active-fixation coronary sinus leads using a mechanical rotation extraction device. Pacing Clin Electrophysiol 2015; 38: 302-5.

20. Lewicka-Nowak E, Kutarski A, Dabrowska-Kugacka A, et al. A novel method of multisite atrial pacing, incorporating Bachmann's bundle area and coronary sinus ostium, for electrical atrial resynchronization in patients with recurrent atrial fibrillation. Europace 2007; 9: 805-11.

21. Lewicka-Nowak E, Dabrowska-Kugacka A, Rucinski P, et al. Atrial function during different multisite atrial pacing modalities in patients with bradycardia-tachycardia syndrome. Circ J 2009; 73: 2029-35.

22. Daubert C, Leclercq C, Pavin D, et al. Biatrial synchronous pacing: a new approach to prevent arrhythmias in patients with atrial conduction block. In: Prevention of Tachyarrhythmias with Cardiac Pacing. Daubert C, Prystowsky E, Ripart A (eds.). Futura Publishing Company Inc., NY 1997; 99-119.

23. Bongiorni MG, Kennergren C, Butter C, et al. The European Lead Extraction ConTRolled (ELECTRa) study: a European Heart Rhythm Association (EHRA) Registry of Transvenous Lead Extraction Outcomes. Eur Heart J 2017; 38: 2995-3005.

24. Kusumoto FM, Schoenfeld MH, Wilkoff BL, et al. 2017 HRS expert consensus statement on cardiovascular implantable electronic device lead management and extraction. Heart Rhythm 2017; 14: e503-51.

25. Bongiorni MG, Burri H, Deharo JC, et al. 2018 EHRA expert consensus statement on lead extraction: recommendations on definitions, endpoints, research trial design, and data collection requirements for clinical scientific studies and registries: endorsed by APHRS/HRS/LAHRS. Europace 2018; 20: 1217. 